training could be improved with requests for a formalised attachment, formal qualification and greater training in practical procedures.

The BASHH GD SIG, in liaison with BASHH, aims to optimise GD training for registrars. Plans for improved resources are in progress, including a practical skills course and e-learning.

\section{P192 SUDDENLY YOU'RE ON YOUR OWN, AND YOU'RE OUT THERE IN THE BIG WORLD: MIDDLE-AGED ADULTS' SEXUAL RISK-TAKING BEHAVIOURS WITHIN THE CONTEXT OF LIFE-COURSE TRANSITIONS}

${ }^{1,2}$ Jenny Dalrymple* ${ }^{1}$ Joanne Booth, ${ }^{1}$ Paul Flowers, ${ }^{1}$ Karen Lorimer. ${ }^{1}$ Glasgow Caledonian University, Glasgow, UK; ${ }^{2}$ NHS Greater Glasgow and Clyde, Glasgow, UK

\subsection{6/sextrans-2015-052126.236}

Background/introduction While sexual activity, including partner change, is known to continue throughout the life course, there is a paucity of qualitative evidence on how adults over 45 years engage with risk for sexually transmitted infections (STIs), limiting the scope for effective health promotion among this age group.

Aims/objectives The research aimed to explore older adults' sexual risk-taking behaviour within the context of sexuality in later life.

Methods A qualitative in-depth study involving 31 interviews with middle aged heterosexual men and women aged 45 to 65 , recruited from sexual health clinic and community settings.

Results Vulnerability to STI risk emerged around key life course transitions, including following divorce, separation and bereavement. Some spoke enthusiastically of embracing sexual freedom and pleasure within a perceived changed culture, resulting in frequent partner change; however, many found themselves 're-engaging' with their sexual careers within an unfamiliar gendered landscape. Lacking an (ageing) body confidence led to the prioritisation of intimacy over STI risk; condoms were viewed as being for birth control and therefore mostly unnecessary, or linked with casual sex and lack of trust. STIs were commonly considered to be a young person's concern.

Discussion/conclusions Information provision alone will not be enough to counter the complexities of navigating the dramatically different sexual landscape these older adults find themselves within compared to their youth, particularly those who have emerged from long-term relationships. A separately focussed approach to STI prevention taking account of life course experience, ageing and cultural change is advocated.

\section{P193 DEVELOPMENT OF A HANDHELD POINT OF CARE MOLECULAR DIAGNOSTIC DEVICE FOR SEXUALLY TRANSMITTED INFECTIONS}

${ }^{1}$ Ruth Mackay*, 1,2Pascal Craw, ${ }^{1}$ Manoharanehru Branavan, ${ }^{3}$ Tariq Sadiq, ${ }^{1}$ Wamadeva Balachandran. 'Brunel University London, Uxbridge, Middlesex, UK; ${ }^{2}$ Environmental Genomics, Oceans and Atmosphere Flagship, Commonwealth Scientific and Industrial Research Organisation, Hobart, Tasmania, Australia; ${ }^{3}$ St. George's, University of London, London, UK

\subsection{6/sextrans-2015-052126.237}

Background/introduction Brunel DoCLab is part of the eSTI2 Consortium which is developing electronic self-testing and portable instruments for sexually transmitted infections using nucleic acid amplification test technologies. We have designed a point of care test platform that integrates a proprietary sample collection device directly with a microfluidic cartridge. A low cost benchtop real-time isothermal amplification platform has been developed capable of running six amplifications simultaneously.

Aim(s)/objectives To evaluate the sample preparation and isothermal amplification within the low cost diagnostic platform. Methods The microfluidic device incorporates passive mixing of the lysis-binding buffers and sample. Cell lysis, within the cartridge, is conducted using a chemical method and nucleic acid purification is done on an activated cellulose membrane. Isothermal amplification was conducted using recombinase polymerase amplification (RPA).

Results Preliminary results have shown extraction efficiencies for this new membrane of $69 \%$ and $57 \%$ compared to the commercial Qiagen extraction method of $85 \%$ and $59.4 \%$ for 0.1 $\mathrm{ng} / \mu \mathrm{L}$ and $100 \mathrm{ng} / \mu \mathrm{L}$ salmon sperm DNA respectively spiked in phosphate buffered solution. Extraction experiments in the passive mixer cartridges with lysis and nucleic acid purification showed extraction efficiency around $80 \%$ of the commercial Qiagen kit. The platform is capable of detecting Chlamydia trachomatis genomic DNA within 10 min using RPA for 100,000 copies $/ \mu \mathrm{L}$.

Discussion/conclusion The work presented here shows a low cost, rapid nucleic acid extraction, isothermal amplification and detection platform for diagnosing C. trachomatis. Work is ongoing to fully integrate the sample-in to result platform for rapid diagnosis of STIs using genital samples.

\section{P194 COST-EFFECTIVENESS OF CHLAMYDIA TESTING IN SCOTLAND}

${ }^{1}$ Lesley Wallace*, ${ }^{2}$ Katharine Looker, ${ }^{2}$ Katy Turner. ${ }^{1}$ Health Protection Scotland, Glasgow, UK; ${ }^{2}$ University of Bristol, Bristol, UK

\subsection{6/sextrans-2015-052126.238}

Background/introduction Scottish chlamydia testing guidelines target symptomatic and high-risk asymptomatic individuals. Recent publications, indicating a low risk of progression to serious chlamydia-related outcomes, particularly tubal factor infertility (TFI), question the validity of high levels of opportunistic testing especially among asymptomatic individuals.

Aim(s)/objectives To examine cost-effectiveness of current chlamydia testing to prevent TFI among those aged 15-24 in Scotland using cost per Quality-Adjusted Life Years (QALYs) gained and to consider alternative testing strategies.

Methods A compartmental deterministic model of chlamydia infection in those aged 15-24 in Scotland was developed to examine the impact of testing coverage and partner notification (PN) on number and cost of TFI cases prevented. Cost-effectiveness calculations were informed by best estimates of the QALYs lost due to TFI.

Results At $16.8 \%$ baseline testing coverage (laboratory data), $4.4 \%$ prevalence (NATSAL-3) and assumed PN rate of 0.4, the total testing cost is $£ 5.4$ million. This is estimated to prevent 258 TFI cases each year in young women. The cost per QALY gained is $£ 40,034$ compared with no testing, using a mid-range health state utility value (HSUV) for TFI $(0.76( \pm 0.24))$ and PID $(0.9( \pm 0.22))$. A $50 \%$ reduction in current testing would result in higher chlamydia prevalence and 84 more TFI cases.

Discussion/conclusion Current chlamydia testing activities in Scotland do not appear cost-effective. However, the model is sensitive to several parameters, particularly the HSUV and there are uncertainties in the current testing costs and progression to 